\title{
GROUNDED THEORY E OS ESTUDOS DE LINGUAGEM: UMA RELEITURA
}

\author{
GROUNDED THEORY AND LANGUAGE STUDIES: A REVIEW
}

Rubens Lacerda de Sá 1

Recebido: 02 fev. 2019

Aceite: 28 mai. 2019

DOI https://doi.org/10.29327/2.1373.1-15

\begin{abstract}
RESUMO: Em tom ensaístico, almejo com este texto obsequiar aos leitores com uma releitura crítica e reflexiva dos princípios norteadores da Grounded Theory e suas potencialidades no campo dos Estudos de Linguagem. Visto que a teoria em tela se arroga como sendo neutra sob os prismas ontológico e epistemológico, penso que um alinhamento com os Estudos de Linguagem pode contribuir para uma melhor (ou outra!?) compreensão do fenômeno que nos propomos a investigar com o fito de aliviar as dores sociais da contemporaneidade. Entendo que toda pesquisa social nasce de uma inquietação, um incômodo, da constatação de dores individuais ou coletivas que operam na tessitura social e que demandam uma intervenção imediata. Enquanto pesquis-a-dor ontológica e socialmente orientado, meu anseio é contribuir para o alívio dessas dores ou "privações sofridas [a fim de] produzir leveza de pensamento e modificar a precariedade da existência" dos atores envolvidos em nossos loci de pesquisa, cf. Rojo (2013, p. 65).
\end{abstract}

PALAVRAS-CHAVE: Grounded theory; Estudos de Linguagem; Releitura.

ABSTRACT: I aim with this essay to provide readers with a critical and reflective review of the guiding principles of Grounded Theory and its potentialities in the field of Language Studies. Once this theory arrogates itself to be neutral under the ontological and epistemological prisms, I think that an alignment with Language Studies can contribute to a better (or other!) understanding of the phenomenon we propose to investigate with a view to soften the social pains of contemporaneity. It's my comprehension that all social research is born of a restlessness, a nuisance, of the finding of individual or collective pains that operate in the social fabric and that demand immediate intervention. As an ontological and socially oriented researcher, my desire is to contribute to the relief of such pains or "deprivations suffered [so as to] produce lightness of thought and modify the precariousness of existence" to the actors involved in our research loci, cf. Rojo (2013, p. 65).

KEYWORDS: Grounded theory; Language Studies; Review.

\footnotetext{
${ }^{1}$ Doutorando em Linguística Aplicada pela Universidade Estadual de Campinas (UNICAMP). Docente, pesquisador e coordenador da Editora do Instituto Federal de São Paulo (IFSP). Líder do Grupo de Pesquisa GIEL/CNPq e pesquisador do CLAEC. E-mail: rubens.sa@,ifsp.edu.br ORCID iD https://orcid.org/0000-0003-2555-0079
} 


\section{O BATE-PAPO INICIAL: ORGANIZAÇÃO DOS DADOS NO CORPUS}

Toda pesquisa social nasce de uma inquietação, um incômodo, da constatação de "privações sofridas" e da "precariedade da existência" individual ou coletiva que opera na tessitura social e que demanda uma intervenção imediata (ROJO, 2013, p. 65). Diante desse mal-estar, o pesquis-a-dor social (SÁ, 2016; 2017) apura evidências que corroboram sua constatação e, em seguida, as organiza sistematicamente para posterior análise e proposição de ações intervenientes. Esse processo validará sua hipótese inicial, singular ou plural, e elevará sua pesquisa a um patamar além de refutação.

Pois bem, entendo que é nesse ponto que esse pesquis-a-dor social organizará os dados do corpus de sua investigação. Convém ressaltar que, para os fins a que destinam essa releitura, ecoo Roland Barthes ao nomear de corpus documental "[...] uma coleção finita de materiais, determinada de antemão pelo analista, com inevitável arbitrariedade, e com a qual ele irá trabalhar." (BARTHES, 1967, p. 96). E, no caso de documentos selecionados virtualmente, McCulloch (2004, p. 29) destaca que essa seleção é tanto arbitrária quanto motivada e finita em sua natureza.

Ao falar sobre a organização desse tipo de corpus, Martin Bauer, do Museu de Ciências Britânico, e Bas Aarts, da Universidade de Londres, dizem que:

Primeiramente, os assuntos devem ser teoricamente relevantes, e devem ser coletados a partir de um ponto de vista apenas. Os materiais têm apenas um foco temático, apenas um tema específico. Em segundo lugar, os materiais devem ser tão homogêneos quanto possível. Isto se refere à substância material dos dados. Em terceiro lugar, um corpus é uma interseção da história. Os materiais a serem estudados devem ser escolhidos dentro de um ciclo natural: eles devem ser sincrônicos (BAUER; AARTS, 2013, pp. 55, 56).

Com esses critérios em mente, posso afirmar que os documentos coletados para uma pesquisa no campo dos Estudos da Linguagem constituem-se tipologicamente em um corpus linguístico. Porém, cabe aqui uma palavra de cautela dos especialistas no que diz respeito a delimitação do tamanho do corpus.

Martin Bauer e Bas Aarts (2013, p. 60) alertam para o fato de que é comum “[...] os pesquisadores coletarem facilmente muito mais material interessante, do que aquele com que 
poderiam efetivamente lidar, dentro do tempo de um projeto". Isso compromete a qualidade do trabalho resultando na superficialidade das análises e falta de clareza ou incongruência nas proposições por conta das restrições de tempo, espaço e outras. Para evitar que isso aconteça, os autores acima sugerem aos pesquisadores que façam “[...] uma avaliação séria e realista dos procedimentos referentes ao tempo exigido para seleção e análise [de seu corpus]" (2013, p. 60).

Sem mais delongas, prossigo para a teoria eleita como objeto central deste ensaio em tom ensaístico e suas potencialidades de aplicação nos Estudos da Linguagem.

\section{TEORIA FUNDAMENTADA}

A Teoria Fundamentada ${ }^{2}$ qual metodologia de pesquisa qualitativa para a organização dos dados de um corpus foi desenvolvida pelos sociólogos Barney Glaser, da Universidade de Columbia, e Anselm Strauss, da Universidade de Chicago. Lucélia Nico menciona as razões pelas quais Glaser e Strauss decidiram desenhar esta teoria à época:

[Barney Glaser], enquanto realizou análises qualitativas, observou a necessidade de realizar comparações entre os dados, a fim de identificar, desenvolver e relatar conceitos. [Anselm Strauss vislumbrava a] necessidade de ir ao campo para descobrir o que ocorria; relevância da teoria, baseada nos dados, para o desenvolvimento de uma disciplina de ação social; complexidade e variabilidade do fenômeno e da ação humana; crença que as pessoas são atores, possuindo um papel ativo em resposta às situações problemáticas; compreensão que as pessoas atuam na base do significado; compreensão de que o significado é: definido e redefinido [na/pela] interação; sensibilidade para o envolvimento e desdobramento com a natureza dos eventos (processo); consciência da interrelação entre condições (estrutura), ação (processo) e conseqüências (NICO, et al, 2007, p. 792).

Posso dizer que essa é a certidão de nascimento da Teoria Fundamentada. Uma de suas características enquanto metodologia qualitativa para o manuseio dos dados de um corpus de pesquisa é que ela se movimenta em um vai e vem indutivo-dedutivo, isto é, ora a organização desses dados se dará a partir da dimensão microtextual em direção à macro, ora vice-versa. Outro

\footnotetext{
2 Adoto o termo Teoria Fundamenta para Grounded Theory por ser o mais amplamente traduzido na literatura científica em língua portuguesa. Essa teoria baseia-se no trabalho de GLASER, B. G.; STRAUSS, A. L. The Discovery of Grounded Theory: Strategies for Qualitative Research. New Jersey: Aldine Transactions, 1967/2006.
} 
aspecto importante da Teoria Fundamentada é que não há uma teoria prévia que a alicerça, ou seja, metodologicamente o pesquisador gera seus dados sem que haja ainda uma teoria anteriormente estabelecida a ser testada. Seus dados após analisados servirão de indicadores e de fundamento para a teoria que deverá ser usada em sua posterior análise. Daí, o nome: Teoria (a ser) Fundamentada.

Em sua obra mais recente, Barney Glaser, um dos fundadores da teoria, voltou a reafirmar o mote central desta assim como ele e seu colega, Anselm Strauss, in memorian, a conceberam:

A Teoria Fundamentada é uma metodologia para a elucidação e geração de teoria diretamente com base nos dados em vez da utilização de uma lógica teórica a partir de uma premissa pressuposta e sua subsequente verificação/teste de hipóteses. Trata-se de evitar todo tipo de interesse profissional preconcebido por parte do pesquisador (incluindo suas preconcepções ontológicas e epistemológicas que são facilmente substituídas por uma atitude pragmática), em favor da descoberta dos interesses daqueles que são seu objeto de estudo. Trata-se de descobrir quais os principais interesses desses. Trata-se de descobrir o que os leva a adotar o comportamento estudado; consequentemente, isso implica na descoberta das soluções mais relevantes e importantes para esses que são objeto de estudo. A Teoria Fundamentada é filosoficamente (quase) neutra. Isso significa que tem nada, ou quase nada, a dizer em termos ontológicos e epistemológicos. Sua perspectiva filosófica se limita aos aspectos da vida social, e como esses podem ser trazidos à baila pela abordagem metodológica da teoria ${ }^{3}$ (GLASER, 2016, pp. 103, 104) ${ }^{4}$

Assim, baseado no mote central da proposta da Teoria Fundamentada ressalto que não se trata de uma teoria para análise de dados e sim para seu tratamento e organização. Nessa releitura da teoria, proponho a organização da mesma em seis estágios em contraponto aos quatro estágios originalmente propostos por Barney Glaser e Anselm Strauss (GLASER; STRAUSS, 1967/2006). A medida que os apresento e argumento, envidarei esforços de indicar as razões para essa releitura e ampliação.

\footnotetext{
${ }^{3}$ The GT perspective is about methodology for discovering and generating theory directly from data instead of logically deriving theory from presupposed premises and subsequent testing/verifying of hypotheses. It is about avoiding all preconceived professional interest concern of the researcher (including preconceived ontological and epistemological assumptions that are best replaced by a pragmatic attitude), in favor of the discovery of the substantive interest concerns of those being studied. It is about the discovery of the main concern of those being studied. It is about what drives and directs the behavior of those being studied; consequently, it will also be the discovery of the most relevant, important and problematic for those being studied and of its recurrent solving. [...] GT is philosophically (almost) neutral. This means that is has nothing or almost nothing to say about ontology and epistemology. Its philosophical stance is limited to the assumption that social life is patterned, and that these patterns can be discovered by the methodological approach of GT.
}

${ }^{4}$ Todas as traduções dos textos originais citados neste manuscrito são de minha autoria e responsabilidade. 
Conjugarei os fundamentos do círculo para compreensão textual propostos na Teoria Hermenêutica-Filosófica (GADAMER, 2002) aos estágios um e dois da Teoria Fundamentada. Em seguida, advogo em favor do auxílio de computador para o processo de organização dos dados dos estágios três, quatro e cinco da Teoria Fundamentada e apresento as vantagens e os potenciais perigos dessa aliança (KELLE, 2013). O sexto estágio da Teoria Fundamentada, em minha releitura, nomeio de triangulação holística dos dados. Esse será alinhavado com os princípios da proposta metodológica de Triangulação Interdisciplinar (JANESICK, 1994). O objetivo nesse estágio é que os dados sejam expostos ao escrutínio de outros investigadores sociais com afiliações epistêmicometodológicas diversas com fito de apontar possíveis direcionamentos teóricos para a análise dos dados. É possível que se chame essa fase de análise dos dados organizados como um sétimo estágio, se assim for preferido, visto que é nesse momento em que os dados triangulados interdisciplinarmente terão indicado as premissas ou pistas epistemológicas a serem usadas no restante da pesquisa.

\subsection{TEORIA FUNDAMENTADA: ESTÁGIOS UM E DOIS}

A citação abaixo sintetiza com clareza os dois primeiros estágios da teoria:

Uma Teoria Fundamentada é aquela derivada indutivamente do estudo do fenômeno que representa. Isto é, ele é descoberto, desenvolvido e provisoriamente verificado por meio de sistemática coleta e análise de dados. Portanto, a coleta de dados, análise e teoria possuem relação recíproca entre si. Não se começa com uma teoria para prová-la. Começa-se com uma área de estudo em que se permite a emersão do que é relevante (STRAUSS; CORBIN, 1990, p. 23).

Em outros termos, primeiro identifica-se o fenômeno-objeto da pesquisa. Em seguida, ele é “descoberto", i.e. um tema, um escopo é delimitado. Logo, os dados são gerados. Nada mais.

A identificação do fenômeno é anterior à pesquisa. É a inquietação, o incômodo, o mal-estar social que move o pesquisador a interessar-se pelo assunto e querer intervir. Isso posto, conjugo esses dois primeiros estágios da Teoria Fundamentada com os norteamentos do círculo para a 
compreensão textual da Teoria Hermenêutica-Filosófica. O objetivo dessa aliança é refinar por completo a delimitação, a "descoberta", do escopo do fenômeno.

\subsubsection{TEORIA HERMENÊUTICA-FILOSÓFICA: ESTÁGIOS UM E DOIS DA} TEORIA FUNDAMENTADA

Hermes, um dos deuses da mitologia grega, possuía inúmeros atributos. Um deles era o de servir como decifrador (em grego: hermeneu) de mensagens ou textos (em grego: tikós) dos demais deuses do Olimpo. Há quem atribua a ele a maestria (em grego: teckhné) com que decifrava suas mensagens. Nasce daí o nome da Teoria Hermenêutica-Filosófica definida como a arte de compreender e decifrar textos ou escritos. Entre seus preceitos estão que essa tarefa é finita e objetiva. Orienta-se prioritariamente por práticas éticas e pela conduta moral.

Hans-Georg Gadamer, autoridade desta proposta metodológica, propõe o conceito do círculo hermenêutico para a compreensão textual dividido em três etapas interligadas, conforme descritas brevemente a seguir (MISSAGIA, 2012). Penso que em uma pesquisa nos Estudos de Linguagem essa mesma subdivisão e procedimentos podem ser usados nos dois primeiros estágios da organização dos documentos do corpus seguindo os passos da Teoria Fundamentada.

A primeira etapa do círculo hermenêutico para a compreensão textual é a pré-compreensão. Esta refere-se à ciência das ideias anteriores ao que se diz, ou seja, o pesquisador sabe que carregará sua própria idiossincrasia, seu ser-estar no mundo.

Gadamer exemplifica essa noção com a leitura de um texto: ao procurar compreender algo que lemos, sempre realizamos um projetar em relação ao sentido do todo, do mesmo modo que partimos das nossas concepções prévias que determinam a compreensão. É somente no confronto com o próprio texto que podemos chegar a questionar esse projetar e os prejuízos que guiaram a leitura. Mas o fato é que uma compreensão prévia do próprio texto já é dada de antemão antes de nossa leitura. Uma estrutura circular análoga estaria presente em qualquer atividade de compreensão (MISSAGIA, 2012, p. 5).

O que se quer dizer é que na seleção dos textos, o pesquisador carregará consigo toda a sua visão de mundo. Trará consigo inclusive aquelas que se relacionam com os critérios que adotará, 
consciente ou inconscientemente, para textos que serão selecionados segundo suas assunções. Por essa razão é que o próprio Hans Gadamer “[...] não pressupõe nem neutralidade com relação à coisa nem tampouco auto-anulamento, mas inclui a apropriação das próprias opiniões prévias e preconceitos.” (GADAMER, 2002, p. 405).

A segunda etapa, do círculo hermenêutico, é uma leitura mais minuciosa e perscrutadora dos textos objeto da investigação. Neste momento, o que se almeja é que o pesquisador social crítico, o hermeneuta, consiga adotar uma postura e uma atitude o mais neutra quanto seja possível, sem envolver-se com o fenômeno ou o material que será objeto de sua compreensão e escrutínio textual.

Para o hermeneuta, não há outro caminho senão assumir e reconhecer explicitamente os prejuízos que são inerentes à sua compreensão prévia: por estarmos inseridos em uma cultura e tempo específicos, devemos reconhecer que nosso ponto de vista é sempre um ponto de vista entre outros, marcado pelos conceitos e paradigmas de nosso contexto. (MISSAGIA, 2012, p. 6).

Mesmo o hermeneuta mais bem intencionado é perpassado, na arena dos discursos, pelas "[...] palavras alheias [ora] contrárias [ora] camaradas [...]" (SOUSA, 2014, p. 20). No entanto, nesta fase de minúcias e aprofundamento intermediário da leitura textual é importante que o pesquisador consiga distanciar-se o máximo que puder de si mesmo para evitar "prejuízos à sua compreensão prévia”. Martin Heidegger, também hermeneuta, dá uma pista para tentar atenuar esse impasse dizendo que isso só será possível por “[...] abandonar o preconceito no momento decisivo, na base do encontro crítico com o assunto em questão. Essa é a forma de existência de um homem de ciencia." (2005, p. 2).

Latorre, Rincón e Arnal (2003) falam do esforço pela compreensão sistemática crítica, objetiva e que prime pelo rigor científico. E Hans Gadamer arremata dizendo que "[...] faz sentido que o [hermeneuta] não se dirija aos textos, a partir de sua opinião prévia que lhe subjaz, mas que examine tais opiniões quanto à sua legitimação, isto é, quanto à sua validez” (2002, p. 403).

Por fim, como parte da terceira etapa do trabalho do pesquisador hermeneuta está a formatação da nova compreensão, ou seja, a (re)formulação da tese inicial ou das intermediárias. Para Hans Gadamer (2002, p. 25) essa só pode acontecer por meio da linguagem, pois “[...] a linguagem enquanto tal é mais do que um comportamento subjetivo". 
O cientista entende que o círculo atinge sua completude somente na esfera discursiva que se processa por meio da linguagem. Essa é que atribui o sentido lato à sua hermenêutica-filosófica.

Do mesmo modo, a ironia, o eufemismo, o sarcasmo e outras figuras de linguagem só são compreensíveis porque o discurso não se resume a proposições rígidas tomadas isoladamente. Gadamer percebeu muito bem a importância dessa esfera comunicativa que transcende a proposição. É fundamental para a hermenêutica tratar dessas questões, pois compreender desse modo mais amplo a comunicação significa ter de tratar do fenômeno da interpretação a partir de uma série de dados que não permitem tomar a obra de arte, o texto, ou o discurso como entidades isoladas (MISSAGIA, 2012, p. 10).

Há por trás de todo esse processo o caráter ontológico e, neste sentido, Lênio Streck foi sagaz ao dizer que "Gadamer eleva a linguagem ao mais alto patamar, em uma ontologia hermenêutica, entendendo, a partir disto, que é a linguagem que determina a compreensão e o próprio objeto hermenêutico" (1999, p. 46) e Manfredo Oliveira sacramenta quando fala que "[...] a linguagem é a nossa morada, porque somos ser-no-mundo; nossa compreensão do mundo é, sempre, linguisticamente interpretada" (1996, p. 216).

Assim, o entendimento de Gadamer de que a compreensão textual plena passaria pelas etapas que descrevi e atingiria seu ápice por meio da linguagem, tornam os princípios do círculo hermenêutico de compreensão textual da Teoria Hermenêutica-Filosófica uma peça importante nos dois estágios iniciais da organização de um corpus de pesquisa.

Portanto, a proposta da aliança que discuto reside justamente no fato de que usando os critérios definidos nas três etapas de leitura do círculo, entendo que possam fornecer pistas que ajudem o pesquisador a perceber o uso que se faz da linguagem e para quais objetivos.

Em linhas gerais, ecoo Gilberto Martins ao dizer que a Teoria Hermenêutica-Filosófica é a “[...] busca do conhecimento através do círculo. Como proposta crítica busca a relação entre o fenômeno e a essência, o todo e as partes, o objeto e o contexto na capacidade de reflexão do pesquisador" (1994, p. 26, 27).

\subsection{TEORIA FUNDAMENTADA: ESTÁGIOS TRÊS, QUATRO E CINCO}




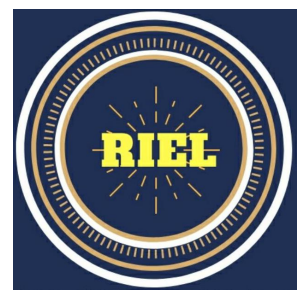

O terceiro estágio inicia com a adoção de critérios de observação rigorosos e sistemáticos, da repetida e constante interação pesquisador-dados. Assim, será possível que esse note a existência de regularidades, ou não, no comportamento do fenômeno que se investiga. Glaser e Strauss (1967/2006, p. 105) chamam esse movimento de "método de comparação constante" em que "[...] o analista inicia por codificar, em tantas categorias quantas forem possíveis, cada incidente que ele observa em seus dados a medida que tais categorias emirjam ou a medida que os dados se encaixam nas categorias ja existentes" 5 . Ampliando o tema, os autores dizem que:

Esta comparação constante dos incidentes logo começam a gerar padrões específicos abrangentes para as categorias. O pesquisador passa a pensar nessas categorias em termos de gerais e em um continuum, dependendo das suas condições de formação, suas implicações, sua relação com outras categorias, e com outros padrões específicos abrangentes ou gerais ${ }^{6}$ (GLASER; STRAUSS, 1967/2006, p. 106).

Trocando em miúdos, os dados passam a ser organizados em categorias que representam “padrões específicos abrangentes/gerais" e estas, por sua vez, são subdivididas "em um continuum" noutras categorias menos abrangentes que os autores chamam de other properties.

Após isso, é hora de avançar para o quarto estágio da organização dos dados quando, segundo Glaser e Strauss (1967/2006, p. 107), “[...] depois de codificar uma categoria umas três ou quatro vezes, o pesquisador talvez comece a ficar mentalmente exausto." 7 e isso poderá acarretar em erros no processo de codificação. Para evitar que isso aconteça, sua sugestão é:

Neste ponto, a segunda regra do método de comparação constante é: pare de codificar e comece a registrar suas ideias em memorandos. Isso servirá para trazer à baila seus pressupostos teóricos iniciais e para aliviar sua mente cansada/em conflito. Ao fazer isso, o pesquisador deverá reservar o tempo necessário para refletir e organizar suas conclusões [até este ponto] e pensamentos de maneira lógica (baseados em dados, não em especulações). [...] Do ponto de vista da

\footnotetext{
5 The analyst starts by coding each incident in his data into as many categories of analysis as possible, as categories emerge or as data emerge that fit an existing category.

6 This constant comparison of the incidents very soon starts to generate theoretical properties of the category. The analyst starts thinking in terms of the full range of types or continua of the category, the conditions under which it is pronounced or minimized, its major consequences, its relation to other categories, and its other properties.
}

${ }^{7}$ After coding for a category perhaps three or four times, the analyst will find conflicts in the emphases of his thinking. 
seleção ou filiação teórica, os memorandos e as notas de campo são geralmente muito úteis ${ }^{8}$ (GLASER; STRAUSS, 1967/2006, pp. 107, 108).

Nesse momento, o pesquisador começa "registrar suas ideias em memorandos e notas de campo". Faz isso, rascunhando possíveis encaminhamentos teóricos que seus dados talvez estejam indicando através das categorias e propriedades, advindas dessas categorias, criadas e organizadas. Digo 'rascunhar' e 'possíveis', pois já há algum encaminhamento teórico prévio, o do pesquisador.

Sobre isso, a ciência crítica discerne que na interação com o fenômeno “[...] não há, nem poderá haver, sensações que não estejam impregnadas de expectativas" (LAKATOS, 1982, p. 15). Esse foi o equívoco inicial de Barney Glaser e Anselm Strauss (1967/2006, p. 37) ao sugerir que o pesquisador estivesse livre de preconcepções teóricas para que sua (nova) "teoria pudesse alcançar uma ampla diversidade de categorias emergentes, sintetizando-as em tantos níveis de generalizações conceituais e hipotéticas quantas fossem possíveis" $"$. Felizmente, em trabalhos posteriores, eles realinharam essa postura um tanto ingênua (STRAUSS; CORBIN, 1990; GLASER, 1978; 1992).

Voltando aos memorandos, Glaser (1978, p. 58) ao apresentar uma de suas vantagens menciona que, nas anotações, "[...] em contraste com uma abordagem generalizadora, uma microanálise por comparação constante, linha a linha, incidente por incidente, é conducente a uma teoria rica e com a sensação de que nada foi esquecido"10. Anselm Strauss e Juliet Corbin (1998, p. 217), acrescentam dizendo que os memorandos "servem de lembretes ou fontes de informação"11 e ampliam sua noção para além de notas de campo. Argumentam que esses são úteis como diretrizes teóricas, lógicas e operacionais e recomendam que os pesquisadores aumentem, de acordo com as categorias codificadas, o nível de complexidade conceitual que está sendo desenvolvido.

\footnotetext{
8 At this point, the second rule of the constant comparative method is: stop coding and record a memo on your ideas. This rule is designed to tap the initial freshness of the analyst's theoretical notions and to relieve the conflict in his thoughts. In doing so, the analyst should take as much time as necessary to reflect and carry his thinking to its most logical (grounded in the data, not speculative) conclusions. [...] From the point of view of generating theory it is often useful to write memos on, as well as code, the copy of one's field notes.

9 [...] theory should aim at achieving much diversity in emergent categories, synthesized at as many levels of conceptual and hypothetical generalization as possible.

10 In opposition to the overview approach, line-by-line or incident-by-incident constant comparative micro-analysis yields a dense, rich theory that gives a feeling that nothing is left out.

$11[\ldots]$ serve as reminders or sources of information.
} 


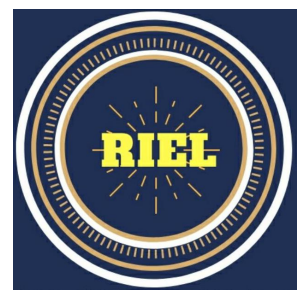

Portanto, nesse estágio, o pesquisador, em suas anotações, começa a perceber as variações nos padrões de regularidade e irregularidade, similaridades e diferenças, a complexidade e densidade das categorias e suas propriedades que emergiram no processo de codificação. De acordo com o contexto de seu fenômeno, inicia o desenvolvimento e o estabelecimento de conceitos importantes que serão muito úteis em estágios posteriores.

Uma vez envolto na dinâmica da Teoria Fundamentada, o pesquisador é automaticamente conduzido do estágio anterior, da criação de conceitos teóricos através de memorandos e notas de campo, ao seguinte, o quinto, a reordenação das categorias e suas propriedades.

Sobre isso, lemos na obra original:

[...] a comparação contínua faz com que o conhecimento acumulado de uma propriedade de determinada categoria comece prontamente a se integrar [com outras], ou seja, passe a estar relacionada de diferentes modos a outras resultando, assim, em um corpo unificado; [...] uma categoria passa a integrar outra categoria de análise 12 (GLASER; STRAUSS, 1967/2006, p. 109).

Esse estágio é necessário porquanto o pesquisador, ao interagir com o fenômeno, quer “[...] responder a perguntas tais como quando, onde, por quê, quem, como, e com que implicações e, desse modo, ampliar seus conceitos de modo contundente"13 (STRAUSS; CORBIN, 1998, p. 125). Anselm Strauss e Juliet Corbin (1998) chamaram esse estágio de codificação axial. Este é mais preciso em relação aos primeiros estágios do processo de organização dos dados na teoria, pois já conta com um eixo central: as categorias e suas propriedades que se encontram sob certa ordenação.

Descrevendo esse processo de reordenação sob eixos temáticos, lemos na obra original:

Este é um método indutivo. Para fazer sentido a partir de tanta diversidade [nas categorias e suas propriedades], o pesquisador é obrigado a desenvolver ideias em um nível de abstração conceitual generalizada maior do que o material qualitativo sob análise. Ele é forçado a conjugar traços uniformes e diversificados subjacentes [em tais categorias e suas propriedades], e usar conceitos mais abstratos para justificar essas diferenças nos dados. Para facilitar o manuseio dos dados, ele é

\footnotetext{
$12[\ldots]$ constant comparison causes the accumulated knowledge pertaining to a property of the category to readily start to become integrated; that is, related in many different ways, resulting in a unified whole; [...] the category becomes integrated with other categories of analysis.

$13[\ldots]$ to answer questions such as when, where, why, who, how, and with what consequences, thus giving the concept greater explanatory power.
} 
obrigado a começar um processo de redução terminológica [de tais categorias e suas propriedades] ${ }^{14}$ (GLASER; STRAUSS, 1967/2006, p. 114).

Partindo da integração e reorganização axial dessas categorias e suas propriedades é possível desenvolver uma tipologia hierárquica. Esta se baseia nos conceitos teóricos do estágio anterior e é chamado de processo de dimensionamento (STRAUSS; CORBIN, 1990, p. 69). Esse processo de dimensionamento, em minha compreensão, é uma releitura da "integração das categorias e suas propriedades" que se funde com a "delimitação da teoria", estágios dois e três respectivamente, na proposta original de Glaser e Strauss (1967/2006, pp. 108, 109).

Talvez sem se darem conta, os próprios autores à época já davam pistas que para o pesquisador essa fusão seria proveitosa visto que "[com ela] fica infinitamente mais fácil lidar com um corpus de dados qualitativos muito grande, [de modo que] o pesquisador pode ficar mais comprometido com as análises tornando-se mais seletivo e focado"15 (GLASER; STRAUSS, 1967/2006, p. 111). Apresentaram ainda outros motivos e o modo como esta fusão deveria ocorrer:

[...] a teoria se estabiliza, no sentido de que as principais modificações vão se escasseando a medida que as comparações das categorias e suas propriedades vão sendo feitas pelo pesquisador. Essas modificações vão ficando cada vez mais no plano de esclarecimento lógico, da retirada de propriedades irrelevantes, da integração de detalhes de algumas propriedades em outras categorias interrelacionadas e, assim a redução vai acontecendo. Por redução, nos referimos às descobertas de uniformidades nas categorias e suas propriedades pelo pesquisador, e que levam à formulação de conceitos teóricos mais concisos. Isto limita a terminologia usada e o tamanho do texto. [Ao mesmo tempo, são mantidas] a parcimônia das variáveis e suas formulações e o escopo da aplicabilidade da teoria em uma variedade de situações, sem perder de foco a relação entre a teoria e os dados 16 (GLASER; STRAUSS, 1967/2006, p. 110).

\footnotetext{
14 This is an inductive method. To make theoretical sense of so much diversity in his data, the analyst is forced to develop ideas on a level of generality higher in conceptual abstraction than the qualitative material being analyzed. He is forced to bring out underlying uniformities and diversities, and to use more abstract concepts to account for differences in the data. To master his data, he is forced to engage in reduction of terminology.

15 [...] increasingly works better for ordering a mass of qualitative data, the analyst becomes committed to [...] analyzing of incidents can become more select and focused.

$16[\ldots]$ the theory solidifies, in the sense that major modifications become fewer and fewer as the analyst compares the next incidents of a category to its properties. Later modifications are mainly on the order of clarifying the logic, taking out non-relevant properties, integrating elaborating details of properties into the major outline of interrelated categories and most important reduction. By reduction we mean that the analyst may discover underlying uniformities in the original set of categories or their properties, and can then formulate the theory with a smaller set of higher level concepts. This delimits its terminology and text. [Keeping] parsimony of variables and formulation, and scope in the applicability of the theory to a wide range of situations, while keeping a close correspondence of theory and data.
} 


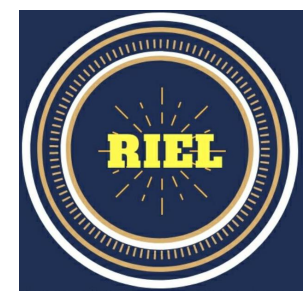

"Os critérios que determinarão a saturação [do processo de dimensionamento] são a combinação dos limites empíricos impostos pelos dados, a integração e densidade teórica e a sensibilidade do pesquisador"17, nos orientam adicionalmente os mentores da teoria, Barney Glaser e Anselm Strauss, (1967/2006, p. 62). É curioso notar como a engrenagem da Teoria Fundamentada vai fazendo com que os dados sejam manuseados e organizados a partir do seu espectro da microrrealidade ao seu contexto macrorreal. Da indução à dedução e, logo, vice-versa.

Os estágios sucintamente apresentados são nevrálgicos na Teoria Fundamentada. Muitos pesquisadores que a adotam como ferramenta metodológica em suas pesquisas qualitativas realizam as tarefas propostas nesses estágios manualmente: com papel e caneta. No entanto, esse trabalho já pode ser feito com a ajuda de programas de computador. Discorro, a seguir, sobre esta aliança.

\subsubsection{AUXÍLIO DE COMPUTADOR: ESTÁGIOS TRÊS, QUATRO E CINCO DA} TEORIA FUNDAMENTADA

É senso comum que o trabalho qualitativo envolve uma enorme tarefa de organização de material. Udo Kelle (2013, p. 395) cita, por exemplo, a quantidade de “[...] transcrições, protocolos, notas, documentos, conceitos teóricos, cadernos, manuscritos, fichas, ideias, argumentações, textos, registros, concordâncias, referências cruzadas, etc." que o pesquisador qualitativo tem de lidar durante as diferentes fases de sua investigação. Por isso, não é de hoje que a tecnologia tem sido uma aliada importante da ciência, como um todo, em seus mais diferentes campos de estudo.

Por exemplo, na área da Tradução, Fábio Alves (2004, p. 185) diz que a parceria se formou na “França e na Rússia nas décadas de 1930 e 1940”. Segundo ele, a partir dessa época e chegando até os nossos dias, têm havido inúmeras "pesquisas que investigam a interface entre o desempenho do tradutor e a tradução assistida por computador".

17 The criteria for determining saturation, then, are a combination of the empirical limits of the data, the integration and density of the theory, and the analyst's theoretical sensitivity. 
A história moderna da Linguística de Corpus remonta a Edward Thorndike que compilou manualmente 4,5 milhões de palavras em uso na língua inglesa e, 25 anos mais tarde, chegou a estonteantes 18 milhões de palavras catalogadas (THORNDIKE, 1921). Em 1953, Michael West lança o General Service List of English Words contendo mais de 2 mil palavras frequentemente em uso em inglês (WEST, 1953). Em 1964, contendo 1 milhão de palavras, a Brown University construiu o primeiro corpora computadorizado. Desde então, conforme nos informa Tony Berber Sardinha (2000, p. 334), “[...] a história da Linguística de Corpus está condicionada à tecnologia, que permite não somente o armazenamento de corpora, mas também a sua exploração [por meio] de ferramentas computacionais para análise de corpus". Assim, esse é outro campo de estudos linguísticos que passou a fazer uso do computador.

Há ainda a Linguística Sistêmico-Funcional que ao conceber a linguagem como sistema sociossemiótico também se vale de recursos computacionais para a organização e análise de seu corpora em determinadas pesquisas (SÁ, 2014). Tem usado com bastante frequência a ferramenta eletrônica chamada Wordsmith Tools que é um recurso tecnológico usado para encontrar padrões lexicais e textuais (SCOTT, 2012).

Eu poderia continuar discorrendo ainda sobre a Linguística Descritiva, a Linguística Computacional, os Estudos do Léxico, a Linguística Aplicada ao ensino de línguas e tantos outros (MATOS, 1989; BRITO, 2000). No entanto, entendo que essa breve introdução já foi suficiente para demonstrar que é histórica a relação entre os estudos linguísticos e o suporte computacional.

Por conseguinte, a medida que essa aliança foi se solidificando, algumas teorias usadas como ferramenta metodológica para o manuseio quantitativo e qualitativo de dados acabaram fornecendo subsídios para o desenvolvimento de vários programas de computadores com o mesmo fim. A Teoria Fundamentada é uma das que subsidiam o desenvolvimento de programas para organização, tratamento e arquivamento de dados em pesquisa qualitativa e quantitativa.

Esses programas de computador passaram a ser usados por pesquisadores do mundo inteiro, e.g. ATLAS.ti ${ }^{18}$ e MaxQDA ${ }^{19}$. Tais programas contemplam quase todas as áreas de pesquisas

\footnotetext{
${ }^{18}$ http://atlasti.com

${ }^{19} \mathrm{http}: / /$ www.maxqda.com
} 


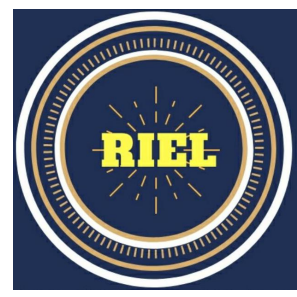

científicas e operacionalizam dados quantitativos. Mas, sobretudo, oferecem excelente suporte para a organização, adequação, catalogação, indexação e muitas outras formas de gerenciamento sistemático de dados qualitativos em formato de textos, gráficos, tabelas, áudio, vídeos, etc.

Udo Kelle nos apresenta algumas "vantagens de softwares para pesquisas qualitativas":

Em primeiro lugar, pelo fato de mecanizar tarefas tediosas e complicadas na organização dos dados, tais como localizar e copiar segmentos de textos. [Ademais], o software ajuda a poupar tempo e pode auxiliar o tratamento de grandes amostras [diante da] quantia de tempo e esforço exigida para preparar os dados. Em segundo lugar, o emprego de pacotes de software pode tornar o processo de pesquisa mais preciso, sistemático e explícito, e por isso mais transparente e rigoroso, pois sistematiza procedimentos que anteriormente eram não sistemáticos, e dando possibilidade aos pesquisadores para documentar exatamente como eles analisam seus dados. [Isso] traz maior confiabilidade [reduzindo] o estilo subjetivo. Em terceiro lugar, o software para [organização e] tratamento de dados textuais pode liberar mais tempo, que pode ser empregado em tarefas mais criativas e analíticas. (KELLE, 2013, pp. 408, 409).

Kelle (2013) chama o processo de organização (manual) dos dados de "tedioso e complicado". Em seguida, alista doze razões para os pesquisadores considerarem o uso de computadores nessa tarefa. Opto por enumerar as vantagens ou razões em pauta: "1) mecaniza as tarefas; 2) localiza segmentos de textos; 3) copia segmentos de textos; 4) poupa tempo; 5) auxilia o tratamento de grandes amostras; 6) [reduz] o esforço exigido para preparar os dados; 7) torna o processo de pesquisa mais preciso, sistemático e explícito; 8) [torna-o mais] transparente e rigoroso; 9) sistematiza procedimentos; 10) documenta exatamente como [o pesquisador] analisa seus dados; 11) traz maior confiabilidade vs. subjetivismo; 12) [propicia tempo para] tarefas mais criativas e analíticas". Não há dúvidas de que há motivos de peso e de sobra para considerar a possibilidade do auxílio de computadores na organização de dados em pesquisa qualitativa.

Por outro lado, estou ciente de que há perigos envolvidos. Alisto, pelo menos, quatro.

O primeiro é que o pesquisador incorre no risco de alienação em relação à materialidade do contexto do fenômeno e os dados gerados à partir desse fenômeno, ou seja, pode ficar tão "encantado" pelos recursos computacionais que perde de vista a inquietação, o incômodo, o malestar inicial. 


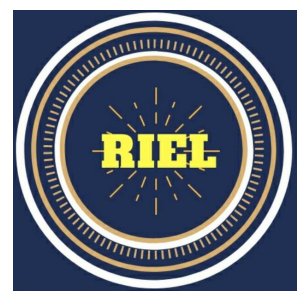

O segundo é que embora se planeje usar o computador como fonte auxílio nessas fases, esse não precisa ser o único método ou técnica a ser utilizada. Sobre isso, Udo Kelle recomenda que essa “[...] escolha pode ser feita tendo em vista o passado metodológico do pesquisador/a, seus problemas de pesquisa, e os objetivos desta pesquisa" (2013, p. 396).

O terceiro perigo é que o pesquisador não deve se esquecer que os computadores:

[...] são instrumentos para mecanizar tarefas de organização e arquivamento de textos, e se constituem em um software para "tratamento e arquivamento de dados", mas não são instrumentos para "análise de dados". Desse modo, a expressão "análise de dados com o auxílio de computador", refere-se à análise interpretativa de dados textuais onde o software é usado para a organizados e tratamento dos dados. (KELLE, 2013, p. 397).

Isto é, nenhum computador será capaz de realizar a compreensão dos sentidos que emanam de determinado texto e, por consequência, realizar a análise per se dos dados. Porquanto, essa é uma tarefa exclusiva do pesquisador.

Por fim, o quarto perigo, maior ainda, foi mencionado por Udo Kelle:

[...] o de uma nova ortodoxia na pesquisa qualitativa [em que] o tratamento dos códigos [ocorre] como se eles fossem representações de uma informação concreta. [Ou seja, há] o perigo de se perder o fenômeno [de vista], devido à reificação dos códigos: o analista começa trabalhando exclusivamente com seus códigos, e se esquece dos dados brutos, sucedendo então que o pré-requisito necessário para que ele pudesse trabalhar, não foi ainda assegurado. [...] Os pesquisadores podem, facilmente, produzir artefatos. (KELLE, 2013, p. 409, 411).

O que se quer dizer é que há o perigo constante de o pesquisador perder o seu referencial. Criar uma "nova ortodoxia" teórico-metodológica. Na Teoria Fundamentada, este deve ser indutivo, interpretativo e com enfoque hermenêutico. Por isso, o pesquisador deve estar atento para não ser seduzido pela função unicamente fatual ao lidar com seus dados. Nessa função o estilo referencial é primariamente dedutivo e clássico.

Em suma, o auxílio do computador pode ocorrer nos estágios três, codificação dos dados do corpus; quatro, criação de memorandos e notas de campos; e, cinco, para o redimensionamento axial dos dados. Retomando a relação computacional com a Teoria Fundamentada, saliento que todo o processo descrito na teoria já pode ser executado hoje por meio de programas de computador 
como os que fiz menção nesta seção, e.g. ATLAS.ti e MaxQDA. Por conseguinte, advogo que o uso desse tipo de programas contribui para o sucesso do trabalho do pesquisador.

\subsection{TEORIA FUNDAMENTADA: ESTÁGIO SEIS}

O dimensionamento axial do estágio anterior é fulcral, pois dá estabilidade ao pesquisador e serve de preparação para o último estágio, o sexto, da organização dos dados do corpus de sua pesquisa. Na obra original Glaser e Strauss chamaram esse estágio de "propor [escrever] uma [nova] teoria"20 (1967/2006, p. 113). Disseram na ocasião:

Quando o pesquisador está convencido de que sua agenda analítica se configura em um corpo teórico bem fundamentado, ou seja, trata-se de um estudo razoavelmente bem embasado do assunto, e que outros na mesma área poderão usar, nesse caso ele deve divulgar os resultados de sua pesquisa com confiança. ${ }^{21}$ (GLASER; STRAUSS, 1967/2006, p. 113).

Foi uma proposta ousada. Ao mesmo tempo, ao ler a obra, este estágio se confunde com o que é genericamente chamado de análise dos dados. Mais tarde, Barney Glaser (1978) falou de desenvolver uma teoria (talvez já existente) que emergisse a partir dos dados. Depois, chamou de agenda teórica (GLASER, 1992). Anselm Strauss e Juliet Corbin (1998) tratam da composição de uma matriz teórica.

A ideia contida no texto original para esse estágio, "propor [escrever] uma [nova] teoria" não é compartilhada pela vasta maioria dos usuários da Teoria Fundamentada hoje. Entretanto, o termo genérico análise de dados é usado por boa parte dos pesquisadores que se valem dessa teoria além de ser usado pelos próprios aios desta, Barney Glaser e Anselm Strauss e, mais recentemente, Juliet Corbin. Eu, particularmente, discordo dessa terminologia.

Discordo por, pelo menos, duas razões. A primeira, é que a Teoria Fundamentada não oferece subsídios para análise per se de dados e sim para organização de dados de um corpus de

\footnotetext{
20 Writing theory.

21 When the researcher is convinced that his analytic framework forms a systematic substantive theory, that it is a reasonably accurate statement of the matters studied, and that it is couched in a form that others going into the same field could use then he can publish his results with confidence.
} 
pesquisa qualitativa. Pelo menos não, quiçá ainda, para os Estudos de Linguagem. E o segundo motivo é porque uma vez findo todo esse processo de organização dos dados e sua visualização holística, por meios diversos, o pesquisador formulará ou será confrontado com uma premissa sócio-epistemológica. Somente, então, é que ele procederá a análise propriamente dita.

Sendo assim, posso asseverar que a Teoria Fundamentada, enquanto ferramenta metodológica indutivo-dedutiva para as pesquisas qualitativas encoraja a teorização heurística e processual. Por heurística, quero dizer que os procedimentos desta teoria contribuem à descoberta por si mesmo, à criatividade em pesquisa. São os dados que indicarão e darão pistas de qual teoria melhor se ajusta ao fenômeno que se observa (GLASER, 1978, p. 36). Não é o pesquisador quem determinará a teoria a que o fenômeno se ajustará, mas o inverso. O fenômeno determinará a teoria.

Por conseguinte, em minha releitura da obra original, esse último estágio deveria ser denominado triangulação holística interdisciplinar dos dados e é descrito da seguinte forma:

Primeiro,

[...] o pesquisador detém a posse dos dados organizados, dos memorandos [e notas de campo], e de uma teoria [a/as do próprio pesquisador]. As discussões [anotações] nos seus memorandos [e notas de campo] delimitaram o conteúdo para o realinhamento das categorias [i.e. dimensionamento axial] que, por sua vez, se converteram em temáticas teóricas mais abrangentes 22 (GLASER; STRAUSS, 1967/2006, p. 113).

Em seguida, nesse processo de rever os dados devidamente organizados e dimensionados axialmente, dá-se início à "criação de uma teoria em desenvolvimento, [ou seja], o nível de generalidade teórica fragmentada pode ser elevada à condição de uma teoria sólida, formal”23 (GLASER; STRAUSS, 1967/2006, p. 114, 115).

Por fim, esse processo de triangulação a que me refiro, sai do nível dos dados e atinge outro patamar, como bem descrevem os preceptores da Teoria Fundamentada:

\footnotetext{
$22[\ldots]$ the analyst possesses coded data, a series of memos, and a theory. The discussions in his memos provide the content behind the categories, which become the major themes of the theory.

$23[\ldots]$ in the creation of a developmental theory ... the level of generality of a substantive theory can be raised to a formal theory.
} 


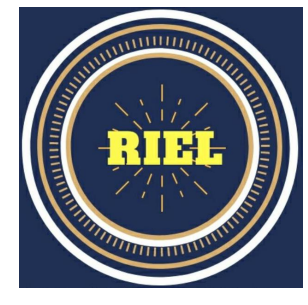

Esse movimento em direção à formalização da teoria exige um escrutínio adicional devido à fragmentação teórica em voga, e o pesquisador deve, conforme já orientamos, incluir em seus estudos material de outras vertentes teóricas. [...] O desenvolvimento de quaisquer perspectivas analíticas coerentes não deve estar restrito única e exclusivamente apenas à uma área específica [Erving Goffman, nota] ${ }^{24}$ (GLASER; STRAUSS, 1967/2006, p. 115).

Aqui, é quando o pesquisador, visando ao "desenvolvimento... de generalidade teórica fragmentada... à uma teoria sólida, formal” triangulará seus dados holisticamente organizados com o "material de outras vertentes teóricas" sem ficar "restrito única e exclusivamente apenas à uma área específica”. Esse tipo triangulação, conforme discuto em detalhes mais adiante, definirá a(s) premissa(s) sócio-epistemológica(s) para posterior compreensão plena do fenômeno em tela.

Logo, o que proponho com isso é uma releitura do último estágio da Teoria Fundamentada que, ao meu ver, é mais coerente com seu próprio objetivo inicial. Isto é, o de formulação heurística e progressiva da(s) teoria(s) de suporte para o posterior estágio de análise.

Disserto, a seguir, acerca da relação entre o estágio seis dessa teoria e a triangulação.

\subsubsection{TRIANGULAÇÃO HOLÍSTICA INTERDISCIPLINAR: ESTÁGIO SEIS DA} TEORIA FUNDAMENTADA

Triangular significa estabelecer as referências, as distâncias e a localização entre pontos. Um exemplo disso são os marinheiros das mais de 1.200 ilhotas que formam as Ilhas Marshall. Eles são capazes de navegar sem a ajuda de mapas e equipamentos de navegação nos quase dois milhões de quilômetros quadrados do oceano Pacífico. Conseguem essa proeza valendo-se de um sistema de triangulação que combina a distância entre a origem e o destino, "[...] varas amarradas a fim de formar uma treliça que representava os padrões das ondas e, por fim, pequenas conchas marinhas eram fixadas para marcar as posições relativas das ilhas" (WATCHTOWER, 2006, p. 32).

Em pesquisa social, muito tem sido dito sobre a importância da triangulação para a validação das análises que são apresentadas nos resultados de uma pesquisa. Do mesmo modo, tem

\footnotetext{
${ }^{24}$ This move to formal theory requires additional analysis of one's substantive theory, and the analyst should, as stated in the previous chapter, include material from other studies with the same formal theoretical import. [...] the development of any one of these coherent analytic perspectives is not likely to come from those who restrict their interest exclusively to one substantive area [Erving Goffman, ft.].
} 
havido muito esforço em busca de um consenso em torno de uma definição e classificação do construto em pauta. Mais do que validar as hipóteses levantadas na pesquisa, Yvonna Lincoln e Norman Denzin dizem que "a triangulação reflete uma tentativa de assegurar uma compreensão em profundidade do fenômeno em questão" (2006, p. 19). Se assumimos que nosso fenômeno é o cerne de nossa pesquisa empírica, a nossa meta será compreendê-lo para transformar sua realidade.

Ainda sobre a importância da triangulação, lemos que esta "é a exposição simultânea de realidades múltiplas, refratadas" (DENZIN; LINCOLN, 2006, p. 20). Sendo assim, o objetivo do pesquisar social deve ser usá-la como um espelho que mostrará os diferentes ângulos de seu fenômeno consolidando suas conclusões ou propondo e indicando novas rotas a serem adotadas.

Zappellini e Feuerschüe (2015) e Fígaro (2014) abordam em seus textos os diferentes tipos de triangulação. Para os fins deste texto considero apenas dois: a triangulação de investigadores e a triangulação teórico-metodológica. Seguindo a proposta metodológica de Valerie Janesick (1994), Donna Towns e Zewelanji Serpell (2004), adoto o conceito de triangulação interdisciplinar.

Alinhado ao estágio seis da Teoria Fundamentada acrescento o termo holístico. Por esse vocábulo, refiro-me a uma visão englobante, completa, múltipla e abrangente de determinado objeto. Assim, o holístico se aplica aos dados de um corpus de pesquisa após organizados e podendo ser visualizados em tabelas, gráficos, grades, mapas mentais, infográficos, textos com anotações marginais, etc. Daí o nome que adoto tanto para esta seção quanto para o estágio seis da Teoria Fundamentada: Triangulação Holística Interdisciplinar.

Tendo explanado sobre o termo holística no nome, espraio-me agora sobre o interdisciplinar.

\section{TRIANGULAÇÃO DE INVESTIGADORES}

Revisito a obra de Barney Glaser e Anselm Strauss (1967/2006) para definição:

Se o pesquisador estiver trabalhando como parte de um grupo de pesquisas, é uma boa ideia discutir suas percepções teóricas com um ou mais membros do grupo. Seus co-pesquisadores podem elucidar alguns pontos que foram deixados para trás, trazer à atenção outros pontos que eles mesmos encontraram em suas próprias 


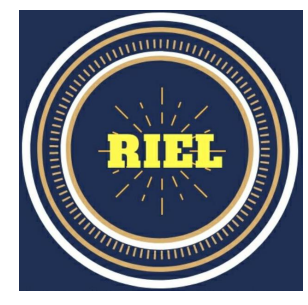

codificações e geração de dados, e fazer uma triangulação de toda essa informação junto com você 25 (GLASER; STRAUSS, 1967/2006, pp. 107, 108).

É bastante suficiente a definição proposta pelos autores para a triangulação de dados por meio de diferentes investigadores. Visto que todos são usuários da linguagem, podem detectar e minimizar eventuais vieses ou tendências do pesquisador. Portanto, neste tipo de triangulação os dados, o cenário, a situação, etc. do mesmo fenômeno são examinados por investigadores diferentes a fim de comparar suas visões sobre o assunto.

Por conseguinte, estou certo de que essa pluralidade de investigadores é útil para que os dados de uma pesquisa sejam observados e pensados com contribuições sob diferentes lentes e perspectivas. Para orientar esse trabalho, sugiro a criação de um roteiro para que os investigadores possam fornecer informações que se alinhem às suas "outras vertentes teóricas" e a pesquisa original não fique "restrita única e exclusivamente apenas à uma área específica" (GLASER; STRAUSS, 1967/2006, p. 115). Nesse roteiro, esses investigadores poderão, e.g. indicar suas impressões sobre o fenômeno em seu aspecto mais abrangente, sua visão sobre os dados e o que esses parecem indicar a partir de seus próprios posicionamentos epistemológicos, que rotas teóricas e ferramental metodológico analítico têm a oferecer e porquê as escolhem, etc.

\section{TRIANGULAÇÃO TEÓRICO-METODOLÓGICA}

Tratando a triangulação sobre o prisma da fusão entre teoria e metodologia, Cresswell e Plano Clark (2013) citam as pesquisas qualiquantitativas com uma abordagem metodológica híbrida mas igualmente passível de triangulação. Zappellini e Feuerschüe (2015, p. 248) se referem “[...] a mobilização de diferentes disciplinas para melhor informar o processo de pesquisa, ampliar o entendimento de um fenômeno e revelar diferentes perspectivas sobre sua natureza".

Na mesma linha, Roseli Fígaro (2014, p. 128) conceitua "triangulação de teoria [como sendo] a abordagem do objeto empírico por perspectivas conceituais e teóricas diferentes. A

\footnotetext{
25 If one is working on a research team, it is also a good idea to discuss theoretical notions with one or more teammates. Teammates can help bring out points missed, add points they have run across in their own coding and data collection, and crosscheck his points.
} 
metodológica quando se utilizam diferentes métodos de investigação para a recolha de dados e a análise do objeto em estudo" (WEYERS; STRYDOM; HUISAMEN, 2011; FLICK, 2009).

Nesse sentido, a fusão entre a teoria e metodologia na triangulação dos dados de um corpus de pesquisa empírica é fundamental. Isto se dá, pois a triangulação sem um ou outro não:

[...] pode se arrogar a pretensão de responder sozinho as questões que a realidade social coloca [Juntos, é possível] iluminar a realidade a partir de vários ângulos, o que permite confluências, discordâncias, perguntas, dúvidas, falseamentos, numa discussão interativa e intersubjetiva na construção e análise dos dados (MINAYO; MINAYO-GOMÉZ, 2003, p. 136).

Depreendo do exposto que a triangulação teórico-metodológica consiste em juntar múltiplas perspectivas teóricas e procedimentos metodológicos de modo interdisciplinar com o fito de atuar em um fenômeno social em comum.

Diego Sousa descreve a concepção de interdisciplinar compartilhada por um grupo de pesquisas brasileiro:

Está relacionada a uma realidade múltipla, diversificada e desterritorializada. Entendemos que sendo interdisciplinar(es), e atuando concordemente, nos apropriamos da universalidade tão própria do fenômeno de língua(gem) transcendendo o espaço físico, abarcando as diversas esferas humanas, territórios do sentido e moradas da linguagem. (SOUSA, 2017, s/n).

Portanto, é totalmente factível pensar em triangulação interdisciplinar visto que os membros desse grupo, usado aqui como exemplo, são filiados a correntes teóricas, com seus pressupostos metodológicos, tão diversas como a Pedagogia Crítica, Estudos do Discurso, Teoria da Complexidade, Interacionismo Sócio-Discursivo, Pedagogia dos Multiletramentos, Estudos Foucaultianos, Teoria Racial Crítica, Estudos de Discurso Críticos, Linguística SistêmicoFuncional, Estudos Literários, Estudos Bakhtinianos e Linguística Aplicada Crítica.

Em conclusão, estou convencido de que o processo de triangulação dos dados do corpus de uma pesquisa será interdisciplinar se contar com os olhares de uma pletora de investigadores e suas múltiplas correntes teórico-metodológicas. Em meio a essa riqueza discursiva, na arena que se formarão, as muitas "palavras alheias [ora] contrárias [ora] camaradas" indubitavelmente indicarão rotas para a análise de dados para que o pesquis-a-dor social possa cumprir seu objetivo derradeiro: 
aliviar as “[...] privações sofridas, produzir leveza de pensamento e modificar a precariedade da existência [...]" dos pesquisados. (SOUSA, 2014, p. 20; ROJO, 2013, p. 65).

Até aqui destaquei os dados de um corpus de pesquisa podem ser totalmente organizados de acordo com os estágios metodológicos da Teoria Fundamentada (GLASER; STRAUSS, 1967/2006) aliados à Teoria Hermenêutica-Filosófica (GADAMER, 2002), ao Auxílio de Computador em Pesquisas Qualitativas (KELLE, 2013) e à Triangulação Interdisciplinar (JANESICK, 1994).

Esses estágios, friso, foram minha releitura baseada principalmente na obra original de Glaser e Strauss (1967/2006). Usei também refinamentos posteriores feitos por Glaser (1978; 1992), Strauss e Corbin $(1990 ; 1998)$ bem como as pesquisas de Kelle (2013), Nico, et al (2007) e Duchscher e Morgan (2004). Conforme mencionei anteriormente, incluo aos estágios descritos na teoria as minhas adaptações visando a contemplar as pesquisas em Estudos de Linguagem.

\section{ARREMATANDO NOSSO BATE-PAPO}

Apresento, na tabela a seguir, um resumo do teor da teoria e minhas considerações. 
Tabela 5 - Teoria Fundamentada: a releitura

\begin{tabular}{|c|c|c|}
\hline $\begin{array}{c}\text { Estágios delineados } \\
\text { na obra original }\end{array}$ & $\begin{array}{l}\text { Releitura e proposta } \\
\text { de novos estágios }\end{array}$ & $\begin{array}{l}\text { Alinhamento com } \\
\text { outras teorias }\end{array}$ \\
\hline- & $\begin{array}{l}\text { 1. Identificação do fenômeno } \\
\text { 2. Delimitação do escopo e tema } \\
\text { do fenômeno }\end{array}$ & $\begin{array}{l}\text { Círculo de Compreensão } \\
\text { Textual: Teoria } \\
\text { Hermenêutica-Filosófica } \\
\text { (GADAMER, 2002) }\end{array}$ \\
\hline $\begin{array}{l}\text { 1. Comparing incidents } \\
\text { applicable to each category }\end{array}$ & 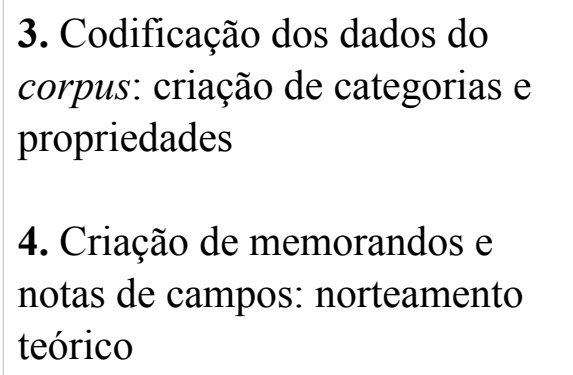 & $\begin{array}{l}\text { Auxílio de Computador em } \\
\text { Pesquisas Qualitativas } \\
\text { (KELLE, 2013) }\end{array}$ \\
\hline $\begin{array}{l}\text { 2. Integrating categories and } \\
\text { their properties } \\
\text { 3. Delimiting the theory }\end{array}$ & $\begin{array}{l}\text { 5. Dimensionamento axial: } \\
\text { integração de categorias e } \\
\text { propriedades e delimitação de } \\
\text { teorias }\end{array}$ & \\
\hline 4. Writing theory & $\begin{array}{l}\text { 6. Triangulação holística } \\
\text { interdisciplinar: definição de } \\
\text { premissas sócio-epistemológicas }\end{array}$ & $\begin{array}{l}\text { Triangulação Holística } \\
\text { Interdisciplinar } \\
\text { (JANESICK, 1994) }\end{array}$ \\
\hline
\end{tabular}

Fonte: Elaborada pelo autor (2019)

Do ponto de vista metodológico, minha releitura da Teoria Fundamentada encerra-se por aqui. Penso que ela cumprirá seu objetivo inicial de entregar os dados de uma pesquisa organizados, triangulados de modo interdisciplinar e com indicadores de premissa(s) sócio-epistemológica(s) tanto a partir de pistas advindas desses dados, pelo olhar do próprio pesquisador, como de coinvestigadores. A etapa seguinte é a que costurará toda a pesquisa: a análise dos dados.

Após essa releitura, penso que é chegado o momento da experimentação. Pretendo aplicar os passos que descrevo e utilizar a cadeia epistêmico-metodológica discorrida neste texto ensaístico 
em um cenário de pesquisa. Almejo, a partir dos resultados dessa experiência, produzir um dossiê temático que incluirá as impressões e as diferentes vozes que se produzirão nas relações dialógicas com os pesquisadores que participarem do processo de triangulação holística interdisciplinar que me refiro no texto em tela. Sendo assim, convido o leitor a continuar na expectativa pelos desdobramentos que se concretizarão na interface Grounded Theory e Estudos de Linguagem.

\section{REFERÊNCIAS}

ALVES, F. Tradução, cognição e tecnologia: investigando a interface entre o desempenho do tradutor e a tradução assistida por computador. In: ROCHA, M.; SPONHOLZ, I.; GERBER, R. M. (Orgs.) Tradução assistida. Cadernos de Tradução, DLLE/UFSC, Florianópolis, Brasil. v. 2, n. 14, 2004, pp. 185-209.

BARTHES, R. Elements of semiology. New York: Hill and Wang, The Noonday Press [tradução do original francês, 1964], 1967.

BAUER, M. W.; AARTS, B. A construção do corpus: um Princípio para a coleta de dados qualitativos. In: BAUER, M. W.; GASKELL, G. (Orgs.) Pesquisa qualitativa com texto, imagem e som: um manual prático. Trad. Pedrinho A. Guareschi. 11a ed. Petrópolis, RJ: Vozes, 2013.

BERBER SARDINHA, A. P. Linguística de Corpus: histórico e problemática. D.E.L.T.A., v. 16, $\mathrm{n}^{\circ}$ 2, 2000, pp. 323-367.

BRITO, G. S. Linguistas e computadores: que relação é essa? Working Papers em Linguística. UFSC, nº 4, 2000, pp. 8-23.

CRESSWEll, J. W.; PLANO CLARK, V. L. Pesquisa de métodos mistos. 2.ed. Porto Alegre: Penso, 2013.

DUCHSCHER, J. E. B.; MORGAN, D.; Grounded theory: reflections on the emergence vs. forcing debate. Journal of Advanced Nursing, 48(6), 2004, pp. 605-612.

FÍGARO, R. A triangulação metodológica em pesquisas sobre a comunicação no mundo do trabalho. Revista Fronteiras - Estudos Midiáticos. 16(2), maio/agosto, 2014, pp. 124-131.

FLICK, U. Introdução à pesquisa qualitativa. 3.ed. Porto Alegre: Bookman, 2009.

GADAMER, H. G. Verdade e método. Trad. Flávio Paulo Meurer. Petrópolis: Editora Vozes, 2002.

GLASER, B. G.; STRAUSS, A. L. The discovery of grounded theory: strategies for qualitative research. New Jersey: Aldine Transactions, 1967/2006. 
. Theoretical sensitivity: advances in the methodology of grounded theory. Mill Valley, CA: The Sociology Press, 1978.

. Emergence vs. forcing: basics of grounded theory analysis. Mill Valley, CA: The Sociology Press, 1992.

The grounded theory perspective: its origin and growth. Mill Valley, CA: Sociology Press, 2016.

JANESICK, V. J. The dance of qualitative research design: metaphor, methodolatry, and meaning. In: DENZIN, N. K.; LINCOLN, Y. S. (Eds.). Handbook of qualitative research. CA: Sage, Thousand Oaks, 1994.

KELLE, U. Análise com auxílio de computador: codificação e indexação. In: BAUER, M. W.; GASKELL, G. (Orgs.) Pesquisa qualitativa com texto, imagem e som: um manual prático. Trad. Pedrinho A. Guareschi. 11a ed. Petrópolis, RJ: Vozes, 2013.

LATORRE, A.; RINCÓN, D.; ARNAL, J. Bases metodológicas de la investigación educativa. Barcelona: Ediciones Experiencia, 2003.

MARTINS, G. A. Manual para elaboração de monografias e dissertações. 3 ed. São Paulo: Atlas, 1994.

MATOS, S. Linguística e informática: perspectivas do uso do computador em Linguística Aplicada e descritiva. Revista FLUP. Universidade do Porto, Porto, Portugal, maio, 1989, pp.117-133.

McCULLOCH, G. Documentary research in education, history and the social sciences. London: Routledge Falmer, 2004.

MINAYO, M. C. S.; MINAYO-GÓMEZ, C. Difíceis e possíveis relações entre métodos quantitativos e qualitativos nos estudos de problemas de Saúde. In: GOLDENBERG, P.; MARSigliA, R. M. G.; GOMES, M. H. A. (Orgs.). O clássico e o novo: tendências, objetos e abordagens em Ciências Sociais e Saúde. Rio de Janeiro: Fiocruz, 2003.

MISSAGIA, J. A Hermenêutica em Heidegger e Gadamer: algumas confluências e divergências. Revista de Filosofia Griot. Amargosa, Bahia, v.6, n. 2, dezembro de 2012, pp. 1-13.

NICO, L. S.; BOCCHI, S. C. M.; RUIZ, T.; MOREIRA, R. S. A Grounded Theory como abordagem metodológica para pesquisas qualitativas em odontologia. Ciência \& Saúde Coletiva, 12(3), 2007, pp. 789-797.

OLIVEIRA, M. A. Reviravolta linguístico-pragmática da Filosofia contemporânea. São Paulo: Edições Loyola, 1996.

ROJO, R. Caminhos para a LA: política linguística, política e globalização. In: NICOLAIDES, C. et al. Política e Políticas Linguísticas. Campinas, SP: Pontes Editores/ALAB, 2013, pp. 63-78. 
SÁ, R. L. Imigração hispano-americana em São Paulo, (des)construção identitária e inclusão dos (in)visíveis: um olhar da Linguística Sistêmico-Funcional. In: III Workshop Systemic Across Languages (SAL). Brasília: Universidade de Brasília, UnB, 2014.

. Içando as velas: uma jornada pro Educ-ação. In: .; et al (Orgs.).

Educação crítica de profissionais da linguagem para além-mar: políticas linguísticas, identidades, multiletramentos e transculturalidade. Campinas, SP: Pontes Editores, 2016.

Imigrantes hispano-americanos, (inter)culturalidade crítica e língua portuguesa.

Revista Estudos Acadêmicos de Letras. UNEMAT. v. 10, nº 01, Julho, 2017, pp. 63-73.

SCOTT, M. Wordsmith tools. Oxford University Press, 2012.

SOUSA, D. P. Vaidade e academicismo: fronteiras epistemológicas. In: V Encontro do Grupo Interdisciplinar de Estudos de Linguagem (GIEL/CNPq): Tabus acadêmicos. Departamento de Educação, Universidade Federal de Lavras. 5. ed., 19/maio. Lavras, MG: DED/UFLA, 2017.

Aquisição dialógica da linguagem: palavra bakhtiniana. 115 f. Dissertação (Mestrado). Cuiabá: Universidade Federal de Mato Grosso, UFMT, 2014.

STRAUSS, A. L.; CORBIN, J. (Eds). Basics of qualitative research: grounded theory, procedures and techniques. Newbury: SAGE, 1990.

Basics of qualitative research: techniques and procedures for developing grounded theory. London: SAGE Publications; 1998.

STRECK, L. L. Hermenêutica jurídica em crise: uma exploração hermenêutica da construção do direito. Porto Alegre: Livraria do Advogado, 1999.

THORNDIKE, E. L. Teacher's wordbook. New York: Columbia Teachers College, 1921.

TOWNS, D. P.; SERPELL, Z. Successes and challenges in triangulating methodologies in evaluations of exemplary urban schools. In: THOMAS, V. G.; STEVENS, F. I. (Eds.). Talent development framework. Hoboken: Wiley Periodicals, 2004.

WATCHTOWER, Bible \& Tract Society of New York, Inc. Ajuda para navegar num vasto oceano. Revista A Sentinela, 1/Out. Brooklyn, Nova Iorque, E.U.A, 2006, p. 32.

WEST, M. A general service list of English words. London: Longman, 1953.

WEYERS, M. L.; STRYDOM, H.; HUISAMEN, A. Triangulation in social work research: The theory and examples of its practical application. Social Work/Maatskaplike Werk, v. 44, n. 2, 2011, pp. 207-222.

ZAPPELLINI, M. B.; FEUERSCHÜE, S. G. O uso da triangulação na pesquisa científica brasileira em Administração. Administração: Ensino e Pesquisa. Rio de Janeiro, v. 16, n. 2, abril-junho de 2015, pp. 241-273. 